\title{
INFRARED OCULOGRAPHY AS A NON INVASIVE METHODS TO MEASURE VISUAL ACUITY BEFORE AND AFTER SURGERY IN CHILDREN WITH CONGENITAL NYSTAGMUS
}

\author{
M.G. Terrin ${ }^{1}$, M. De Berardinis ${ }^{1}$, D. Boccuzzi ${ }^{1}$, G. Terrin ${ }^{2}$, A. Magli ${ }^{1}$ \\ ${ }^{I}$ Department of Ophtalmology, University of Naples 'Federico II', Naples, ${ }^{2}$ Women Health, University of \\ Rome "La Sapienza", Rome, Italy
}

Background: Congenital nystagmus is an involuntary, bilateral, conjugate oscillation of the eyes which is present at birth or develops within the first 6 months of life. A specific surgical intervention may improve visual acuity in these subjects. However, measurement of visual acuity in children is difficult because of invasive procedures and scarce patient's collaboration. Infrared oculography (IROG) is a non invasive method validated to define time of foveation, an indirect measure of visual acuity.

Aims: To evaluate the utility of IROG in children with congenital nystagmus treated by surgery. Methods.
Prospective study enrolling children with congenital nystagmus and anomalous head position. All subjects
underwent surgical intervention according to Anderson. Before and after surgical intervention visual acuity
was measured by IROG.

Results: We enrolled 18 subjects with congenital nystagmus (Age $12.1 \pm 6.6$ y, Male 11) and anomalous right $(n=9)$ or left $(n=9)$ head position. After surgery, head position was corrected in 9 out of 18 subjects. Visual acuity measured by IROG improves in all enrolled subjects after surgery (NAFP $0.68 \pm 0.07$ before vs $0.73 \pm 0.04$ after surgery). IROG was well tolerated by all enrolled subjects.

Conclusions: IROG is an useful non invasive tool to evaluate efficacy of surgery for congenital nystagmus in pediatric patients. 